\title{
"Section to Point" Correction Method for Wind Power Forecasting Based on Cloud Theory
}

\author{
Dunnan Liu, ${ }^{1}$ Yu Hu, ${ }^{1}$ Yujie $\mathrm{Xu},{ }^{1}$ and Canbing $\mathrm{Li}^{2}$ \\ ${ }^{1}$ School of Economics and Management, North China Electric Power University, Beijing 102206, China \\ ${ }^{2}$ College of Electrical Engineering, Hunan University, Changsha 410006, China \\ Correspondence should be addressed to Yu Hu; huyu0728@qq.com
}

Received 11 September 2014; Revised 11 November 2014; Accepted 21 November 2014

Academic Editor: Davide Spinello

Copyright (c) 2015 Dunnan Liu et al. This is an open access article distributed under the Creative Commons Attribution License, which permits unrestricted use, distribution, and reproduction in any medium, provided the original work is properly cited.

\begin{abstract}
As an intermittent energy, wind power has the characteristics of randomness and uncontrollability. It is of great significance to improve the accuracy of wind power forecasting. Currently, most models for wind power forecasting are based on wind speed forecasting. However, it is stuck in a dilemma called "garbage in, garbage out," which means it is difficult to improve the forecasting accuracy without improving the accuracy of input data such as the wind speed. In this paper, a new model based on cloud theory is proposed. It establishes a more accurate relational model between the wind power and wind speed, which has lots of catastrophe points. Then, combined with the trend during adjacent time and the laws of historical data, the forecasting value will be corrected by the theory of "section to point" correction. It significantly improves the stability of forecasting accuracy and reduces significant forecasting errors at some particular points. At last, by analyzing the data of generation power and historical wind speed in Inner Mongolia, China, it is proved that the proposed method can effectively improve the accuracy of wind speed forecasting.
\end{abstract}

\section{Introduction}

Wind power is a critical component of new energy. It can be grid connected with some advantages like safety, reliability, nonpollution, and being fuel-free, which has undergone rapid growth worldwide in recent years. China also attaches great significance to the development of wind energy resources. However, as the scale of wind power keeps expanding, the large scale of wind power grid connected proposed a severe challenge to the security and stability of the grid because of the constant changing of wind output with wind speed. In order to improve the reliability of wind power consumption, the accuracy of wind power prediction is very important [1].

There are mainly two wind power forecasting methods at present. One is to forecast wind power by wind speed according to the wind power formula [2-4]. The other is to make statistical prediction based on historical data by adding meteorological factors (wind speed, wind direction) as auxiliary forecasting $[5,6]$. Regarding the first method, the wind power depends on the wind speed of wind power station, which is mainly influenced by the forecasting accuracy of wind speed. However, the wind speed is changing irregularly and the wind power and wind speed are both quantitative data. It is difficult to establish the exact correlation between the wind power and wind speed. In the second method, the overreliance on historical data leads to the lower forecasting accuracy when the catastrophe point occurs.

Most of the current methods can only forecast the "point to point" wind power. These methods establish a causal model between the wind power and wind speed about each point in time based on historical data, that is, to forecast the wind power at particular time point according to the wind speed of the same time point.

References $[7,8]$ forecasted the wind speed of wind turbines based on physical method with the data of wind speed and its direction, taking into account topographic change and wake effect. And the method proposed is capable of forecasting the wind power output by considering wind power curve. However, it has significant influence on the wind power forecasting accuracy, because some numerical weather prediction (NWP) values cannot provide accurate values at some catastrophe points. It has significant influence on the wind power prediction accuracy. Reference [9] derived the causality equation between the wind speed and wind 
power based on the grey theory. Similarly, this kind of approach also leads to a significant error at the catastrophe points. By building autoregressive moving average (ARMA) model to predict wind speed, the paper results show that when the wind speed experienced a large shock, the deviation shocks accordingly. Besides, the longer the forecasting, the greater the deviation. So this method is more suitable for the ultra-short-term wind speed forecasting [10]. References [11-13] established the association between the wind speed and wind power through intelligent algorithms. The results show that the forecasting accuracy is higher for a smooth sequence, while forecasting accuracy is not very satisfactory when data catastrophe points occur. In the "point to point" approach proposed above we usually present different degrees of trend inertia when forecasting the catastrophe points, which therefore affect the forecasting accuracy.

Therefore, there are mainly two ways to improve the accuracy of wind power forecasting, which is of great significance: first, how to establish an accurate association between wind speed and wind power; second, how to ensure the stability of the catastrophe points forecasting.

To this end, we use the "section to point" correction method of the wind power forecasting model to improve the forecasting accuracy. Firstly, the improved correlation between the wind speed and wind power is presented. To a large extent, the stability of forecasting accuracy can be greatly enhanced by reducing errors at catastrophe points.

\section{Cloud Theory}

2.1. Cloud Model. Dr. Li has proposed a cloud that depicts uncertainty concept of natural language on the basis of randomness and fuzziness [14]. It makes uncertain transformation between qualitative concept expressed by natural language and quantitative expression.

If $U$ is a quantitative universe represented with an accurate value, $C$ is a qualitative concept in $U$, and quantitative value $x \in U, x$ is a stochastic realization of qualitative concept $C$, and the certainty degree of $X$ to $C$ is a random number with stability. Then, the distribution of $X$ in universe $U$ is called Cloud.

First, all maps from $x \in U$ to interval $[0,1]$ are a one-tomany transformation. The membership degree of $x$ to $C$ is a probability distribution but not a fixed value, whose graphic looks like a cloud rather than a distinct curve. Second, cloud consists of lots of droplets, and every droplet is a quantitative concept transformed from a qualitative concept. One droplet may be insignificant, but specification of cloud at different times may make a difference. Therefore, the whole figure of the cloud reflects quantitative concept characteristics. Similarly, the distribution of droplets looks like clouds in the sky, and we cannot see clear boundary nearby but we can see a cloud at distance. That is why we name the figure as cloud. Third, Mathematical Expected Curve of a cloud is its membership degree curve from the point of fuzzy set theory. Fourth, the thickness of the cloud is uneven. It is thicker at waist because droplets are scattered, while it is thinner at top and in the bottom, because droplets are concentrated there. The thickness of cloud reflects the randomness of membership degree. Thus, close to or far from the concept center means the randomness of the membership degree is small, while, as for droplets neither near nor far from concept center, the randomness of the membership degree is big.

Cloud is an uncertain transformation model between qualitative concept and quantitative value. Cloud model generally uses three numerical characteristics, Expectation Ex, Entropy En, and Excess Entropy He, to represent a concept as a whole, as shown in Figure 1.

Expectation Ex: it is the expectation of cloud entropy in domain space. Generally speaking, it is a dot that can best represent qualitative concept or a most typical sample of quantified concept.

Entropy En: it is the uncertain measure of qualitative concept. When the entropy is larger, the numerical range that can be accepted by the concept becomes larger, which means the concept is vaguer.

Hyper Entropy $\mathrm{He}$ is entropy of Entropy En, which reflects dispersion of droplets. The larger Hyper Entropy He is, the larger its dispersion, membership degree, and thickness are.

We can see that the fuzziness and randomness are integrated by three digital features of cloud model into mutual maps of qualitative and quantitative concepts.

Normal cloud model is generally applied to express language value; its Mathematical Expected Curve is as follows:

$$
\operatorname{MEC}_{A}(x)=e^{-\left((x-E x)^{2} / 2 \mathrm{En}^{2}\right)} .
$$

Generation algorithm of normal cloud is as follows:

(1) $x_{i}=G(\mathrm{Ex}, \mathrm{En})$, generating normal randomness $x_{i}$, which takes Ex as its expectation and En as its standard deviation;

(2) $\mathrm{En}_{i}^{\prime}=G(\mathrm{Ex}, \mathrm{He})$, generating normal randomness $\mathrm{En}_{i}^{\prime}$, which takes En as its expectation and Hen as its standard deviation;

(3) calculate $\mu_{i}=e^{-\left(\left(x_{i}-\mathrm{Ex}\right)^{2} / 2 \mathrm{En}_{i}^{\prime 2}\right)}$, and take $\left(x_{i}, u_{i}\right)$ as droplets.

If three eigenvalues (Ex, En, He) of normal cloud is given. Above algorithm can be applied to generate normal cloud consists of an arbitrary number of droplets. Cloud generated by this algorithm normally has uneven thickness, so these three eigenvalues can depict the whole figure of the cloud and no more definition is required to define waist, top and bottom of the cloud.

2.2. Cloud Generator. Generation algorithm can be realized not only by software, but also by hardware, which is named cloud generator; four cloud generators are proposed in this paper.

2.2.1. Forward Cloud Generator. It is a map from qualitative aspect to quantitative aspect, which is on the basis of the numerical characteristics of the cloud (Ex, En, He). Some 2$d$ points-cloud droplets $\operatorname{drop}(x, y)$ of normal cloud model is generated. As shown in Figure 2. 


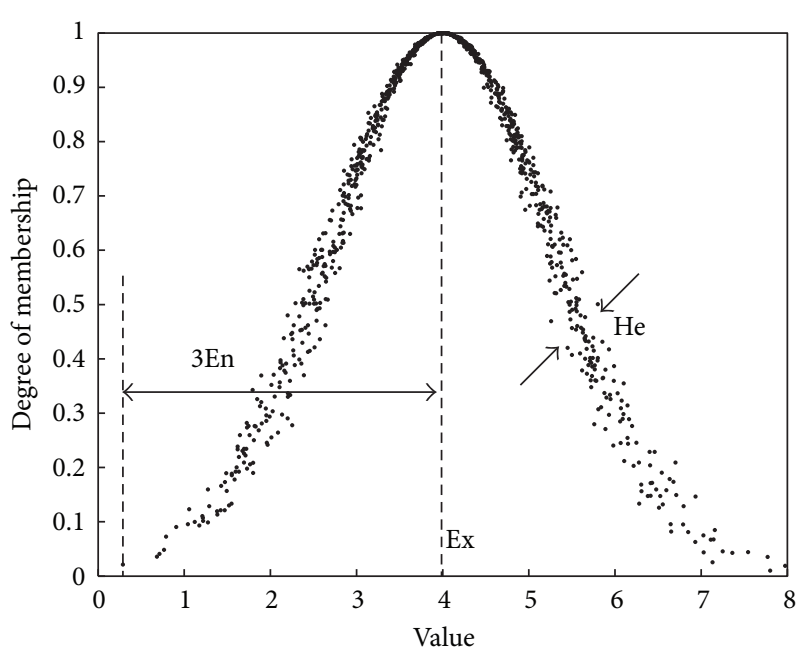

FIGURE 1: Cloud numerical characteristics.

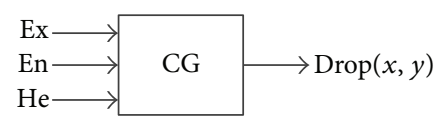

FIgURE 2: The schematic diagram of forward cloud generator.

Following is the algorithm.

Input. The numerical characteristics (Ex, En, $\mathrm{He}$ ) of $1-d$ qualitative concept and the number of cloud droplets $N$.

Output. Quantitative value $x$ of $N$ cloud droplets and the certainty degree $y$ of the concept.

(1) Generate a normal random number En', which takes En as expectation and $\mathrm{He}$ as average variance.

(2) Generate a normal random number $x$, which takes En as expectation and $\mathrm{En}^{\prime}$ as average variance.

(3) Calculate certainty degree

$$
y=e^{-\left(x-E_{x}\right)^{2} / 2\left(E_{n}^{\prime}\right)^{2}} .
$$

(4) $X$ with the certainty degree $y$ becomes a cloud droplet in the universe.

(5) Repeat steps (1) to (4), until generating $n$ cloud drops required.

2.2.2. Backward Cloud Generator. It is a model that realizes conversion from quantitative value to qualitative concept. It can convert an amount of accurate values to qualitative concepts represented by the numerical characteristics (Ex, En, He), as shown in Figure 3.

Following is the algorithm (a backward algorithm without need of the certainty degree information).

Input. Sample dot $x_{i}$, where $i=1,2, \ldots, n$.

Output. The numerical characteristics (Ex, En, He) which reflect qualitative concept.

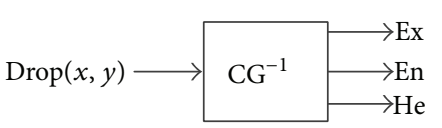

FIGURE 3: Schematic diagram of backward cloud generator.

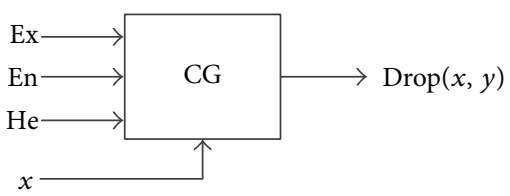

FIGURE 4: Schematic diagram of $X$-conditional cloud generator.

(1) On the basis of $x_{i}$, sample average of this set of data is calculated, as the following equation shows:

$$
\bar{X}=\frac{1}{n} \sum_{i=1}^{n} x_{i} .
$$

(2) Then calculate the first-order central absolute moment $\bar{X}^{\prime}$ as follows:

$$
\bar{X}^{\prime}=\frac{1}{n} \sum_{i=1}^{n}\left|x_{i}-\bar{X}\right| .
$$

The sample variance $S^{2}$ is as follows:

$$
S^{2}=\frac{1}{n-1} \sum_{i=1}^{n}\left(x_{i}-\bar{X}\right)^{2} \text {. }
$$

(3) The expected value is calculated as $\operatorname{Ex}=\bar{X}$, entropy as $\mathrm{En}=\sqrt{\pi / 2} \times \bar{X}^{\prime}$, and excess entropy as $\mathrm{He}=$ $\sqrt{S^{2}-E^{2}}$.

2.2.3. X-Conditional Cloud Generator. Given a quantitative value $x$ in the universe, through forward cloud generator, we can get a certainty degree $y$, which is the qualitative concept of quantitative value $x$, as shown in Figure 4.

Following is the algorithm.

Input. The numerical characteristics ( $\mathrm{Ex}, \mathrm{En}, \mathrm{He})$ of an amount of qualitative concepts and quantitative value $x$.

Output. The certainty degree $y$, the qualitative concept of quantitative value $x$.

(1) Generate normal random number $\mathrm{En}^{\prime}$, which takes En as the expectation and He as average variance.

(2) Calculate the certainty degree as (2).

2.2.4. Y-Conditional Cloud Generator. Given a certainty degree $y, y \in[0,1]$. Through normal cloud generator, we can get a quantitative value $x$ that can conform to the certainty degree $y$ in qualitative concept. The certainty degree $x$ carries uncertainty every time, as shown in Figure 5. 


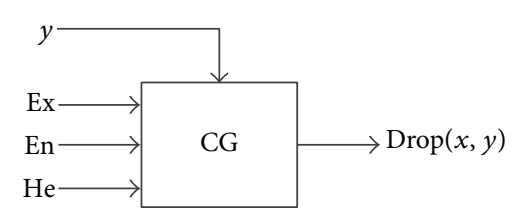

FIGURE 5: Schematic diagram of $Y$-conditional cloud generator.

Following is the algorithm.

Input. The numerical characteristics (Ex, En, He) of an amount of qualitative concepts and certainty degree $y, y \in$ $[0,1]$.

Output. The certainty degree $y$, the qualitative concept of quantitative value $x$.

(1) Generate forward random number $\mathrm{En}^{\prime}$, which takes En as expectation and $\mathrm{He}$ as average variance.

(2) Calculate quantitative value $x=\operatorname{Ex} \pm \operatorname{En}^{\prime} \times \sqrt{-2 \ln y}$.

2.3. Rule Generator. Single condition and single rule generator can be formally represented as

$$
\text { If } A \text { then } B \text {, }
$$

where $A, B$ are qualitative concepts. Take the rule "If wind speed is fast, then generated power is high" as an example; $A$ represents qualitative concept "fast wind speed," and $B$ represents qualitative concept "high generated power." Connect an antecedent cloud generator with a subsequent cloud generator as shown in Figure 6; a single condition rule is constructed.

\section{3. "Section to Point" Correction Method for Wind Power Forecasting Based on Cloud Theory}

First of all, the quantitative values of the wind speed and wind power are converted to qualitative concepts which can be expressed by nature language based on cloud transformation and backward cloud generator of cloud theory [15]. Then, multiple qualitative rules and corresponding reasoning mechanism are constructed, and the wind power eigenvalue which represents the characteristics of current cycle can be obtained. Second, current cloud and historical cloud which have the highest similarity with the current cloud are integrated to create a new cloud through cloud synthesization. Then, reasoning mechanisms between adjacent time sections are established, and the "section to point" correction coefficient is concluded. The corrected forecasting can be obtained through the wind power eigenvalue $P$ and correction coefficient $C$. The forecasting process is shown in Figure 7.

In this paper, the principle of improving forecasting accuracy is mainly reflected by the following two aspects.

(1) Based on cloud theory, enhance the relevance between data and decrease the information loss.

According to two consecutive years of Inner Mongolia Wind Power sampling data, this paper selects twenty data

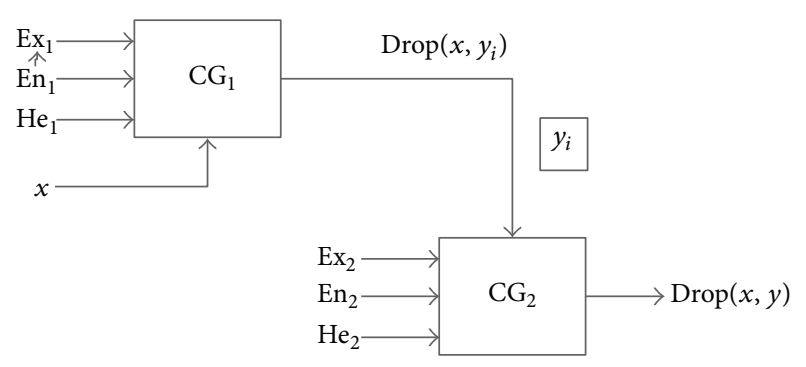

FIGURE 6: Rule generator.

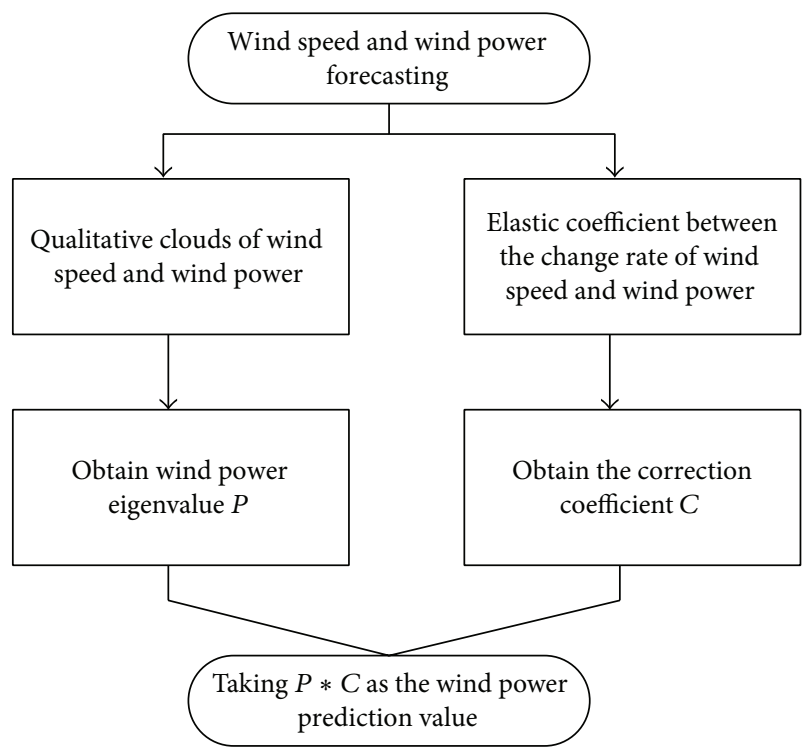

FIGURE 7: Flowchart of wind power forecasting.

dots to calculate the wind speed forecasting value and the wind power value, respectively. As shown in Figure 7, the change of relative error of the wind speed has significant impacts on predicted value of the wind power. It shows that the uncertainty of the wind speed is the main reason for undesirable accuracy of the wind power.

As quantitative data, if the reasoning formula between the wind speed and wind power is deduced by numeric values, correlation results will be inaccurate under the influence of invisible factors. In this paper, quantitative and qualitative data can be transformed effectively based on cloud theory. Then accurate association rules considering invisible factors are constructed by data mining. In this paper, the construction of forecasting model and the calculation of the "section to point" correction coefficient are both based on cloud theory. This is the guarantee of improving prediction accuracy in this paper.

(2) With the help of change discipline of the adjacent time, the "section to point" correction can slow down tendency inertia caused by the wind speed catastrophe.

Processed by cloud synthesization, the wind speed and wind power data contain both the characteristics of current trends and change laws of historical data. Based on this, 


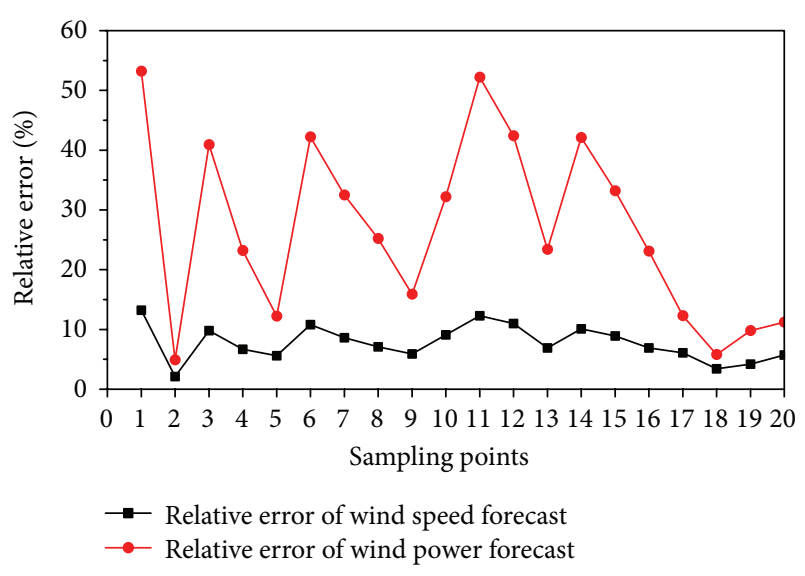

FIGURE 8: Prediction comparison of relative error between wind speed and power.

correction coefficient calculated by this model can restrain prediction fluctuation and significantly improve forecasting, especially when the wind speed changes abruptly. Like a "sword," the "section to point" correction in this paper can improve forecasting accuracy effectively.

3.1. Wind Power Eigenvalue Forecasting Based on Cloud Theory. First, transform [16] the wind speed and wind power data to multiple qualitative concepts, which are used for concepts retrieval [17]. Then, mine association rules between cloud concepts. At last, cloud reasoning [18] model is used to obtain forecasted values. Figure 9 shows the forecasting procedures.

3.1.1. Cloud Transformation and Concept Retrieval. The first step of the wind power forecasting consists of cloud transformation and concept abstracting. Then, cloud generator is built based on association rules mining from similar cloud concepts. So, wind power eigenvalue can be forecasted after inputting the wind speed data.

3.1.2. Setting Up Cloud Generator. The $X$-condition cloud is applied to input $x$, which is the nearest point to forecasted value, and get the membership degree $y_{i}(i=1,2, \ldots, 7)$. Then, take the cloud $\mathrm{CG}_{i}$ and its corresponding rule represented by max $y_{i}$ as forward and backward clouds, which comprise the $Y$-condition cloud to get the forecasting value $m$. The rules generator is shown in Figure 10.

3.2. The Principle of "Section to Point" Correction. The principle of "section to point" correction is correcting the forecasted value of the wind power by cloud model, through synthesization of change rules between wind speed and wind power.

Data near the forecasting point is taken as basic sequence in this paper. The step is taken as $n$, which means this basic sequence's counts are " $n$." Then a sequence of the highest similarity is selected through historical data, and the two sequences are integrated by cloud synthesization. Formula (7) shows the algorithm of cloud synthesization, and results are shown in Figure 11:

$$
\begin{gathered}
\mathrm{Ex}=\frac{\mathrm{Ex}_{1} \mathrm{En}_{1}+\mathrm{Ex}_{2} \mathrm{En}_{2}}{\mathrm{En}_{1}+\mathrm{En}_{2}}, \\
\mathrm{En}=\mathrm{En}_{1}+\mathrm{En}_{2}, \\
\mathrm{He}=\frac{\mathrm{He}_{1} \mathrm{En}_{1}+\mathrm{He}_{2} \mathrm{En}_{2}}{\mathrm{En}_{1}+\mathrm{En}_{2}} .
\end{gathered}
$$

As can be seen from Figure 8, the range of the basic cloud is between 0 and 8 ; therefore forecasting value will be located between 0 and 8 based on basic cloud. If the membership degree of current wind speed in basic cloud is taken as 0.8 , the corresponding value is $4.4(\mathrm{~m} / \mathrm{s})$ as shown in Figure 8 . After combining with historical cloud, the value changes to $7.3(\mathrm{~m} / \mathrm{s})$ in synthesized cloud, but the membership degree is less than 0.1 in basic cloud with the wind speed of $7.3(\mathrm{~m} / \mathrm{s})$. And the tendency inertia of catastrophe can be reduced by using synthesized cloud.

As shown in Figure 12. The forecasted value will not only contain the trend of adjacent wind speed sequence, but also contain change laws of historical cloud. It equals correcting a forecasting value through historical data in a period of time, which is referred to as "section to point" correction in this paper.

3.2.1. Data Processing. Before correction, the data of the wind speed and wind power must be processed as follows.

(1) The wind speed change rate: the formula is shown as follows:

$$
Q_{t}=\frac{V_{t+1}-V_{t}}{V_{t}}
$$

In formula (8), $V_{t}$ means the wind speed at the time point $t$. $V_{t+1}$ means the wind speed at the next point.

(2) Elastic coefficient, which means the effect of the wind speed while the wind power changes: the formula is shown as follows:

$$
K_{t}=\frac{\left(P_{t+1}-P_{t}\right) / P_{t}}{\left(V_{t+1}-V_{t}\right) / V_{t}}
$$

In formula (9), $P_{t}, P_{t+1}$ means the wind power in the time point $t$ and the next point $t+1$.

(3) Divide historical data.

A sequence is constructed according to these two types of data mentioned based on the historical data:

$$
X=\left(K_{1}, K_{2}, \ldots, K_{t}\right) \text {. }
$$

$m$ means the time span value; then we can convert $X$ to $Y$, which is composed of $m$-dimensional vector, and the counts are $t-m+1$; formula (11) is shown as follows:

$$
Y=\left[\begin{array}{cccc}
K_{1} & K_{2} & \cdots & K_{m} \\
K_{2} & K_{3} & \cdots & K_{m+1} \\
\vdots & \vdots & \cdots & \vdots \\
K_{t-m+1} & K_{t-m} & \cdots & K_{t}
\end{array}\right]
$$




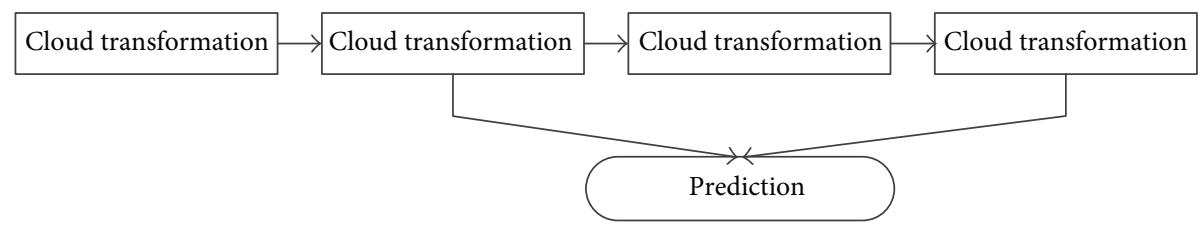

Figure 9: Flowchart of power eigenvalue prediction.

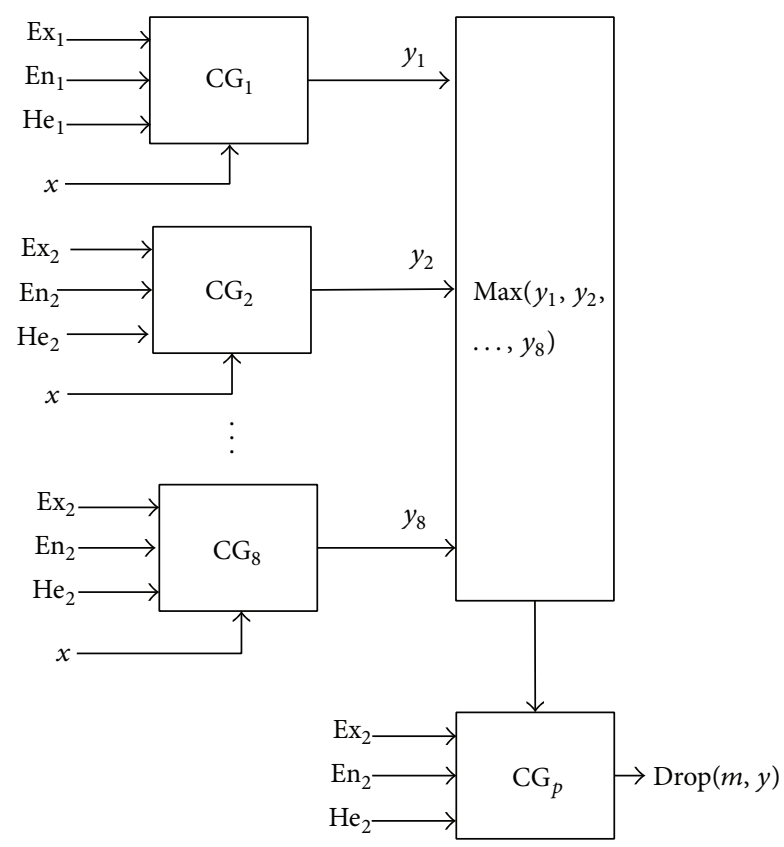

FIgURE 10: Rules of cloud reasoning

3.2.2. Correction Step. The process of "correction step" are shown in Figure 13.

(1) Through backward cloud generator, three characteristic numbers $\left(\mathrm{Ex}_{1}, \mathrm{En}_{1}, \mathrm{He}_{1}\right)$ in current cloud are generated by the present sequence $X_{n}$.

(2) Then, three characteristic numbers $\left(\mathrm{Ex}_{2}, \mathrm{En}_{2}, \mathrm{He}_{2}\right)$ are generated by the historical sequence $Y_{n}$ which has the highest similarity with $X_{n}$. The theory of similarity comparison is as follows.

If current vector is $\alpha=\left(x_{1}, x_{2}, \ldots, x_{m}\right)$ and historical vector is $Y_{i}$, which means the $i$-dimension vector for $Y$, it is recorded as $\beta, 1 \leq i \leq t-m+1$. The formula is shown as follows:

$$
\begin{gathered}
r_{\alpha \beta}=\frac{\alpha \beta}{|\alpha| \cdot|\beta|}, \\
|\alpha|=\sqrt{\sum_{i=1}^{m} x_{i}^{2}}, \quad i=1,2, \ldots, m .
\end{gathered}
$$

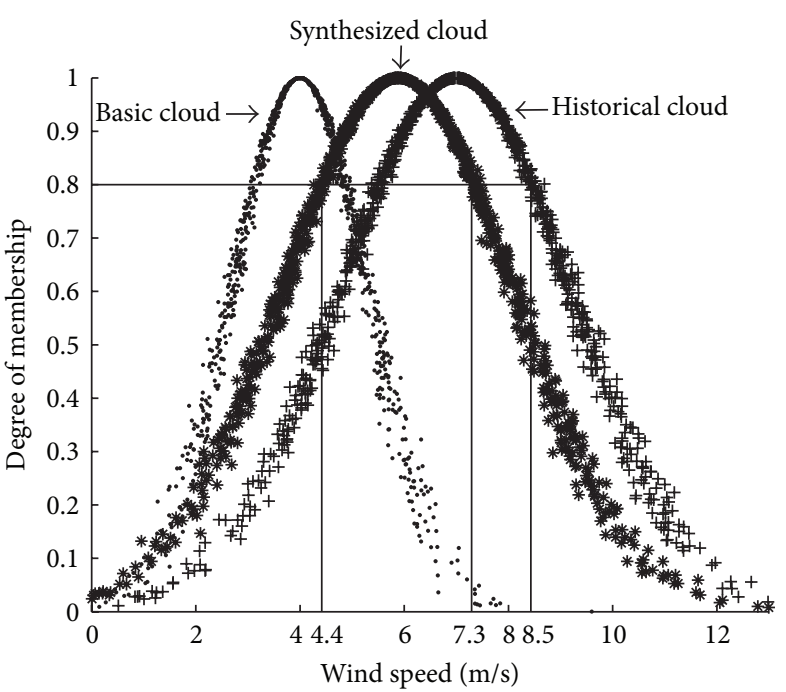

FIGURE 11: Cloud synthesization.

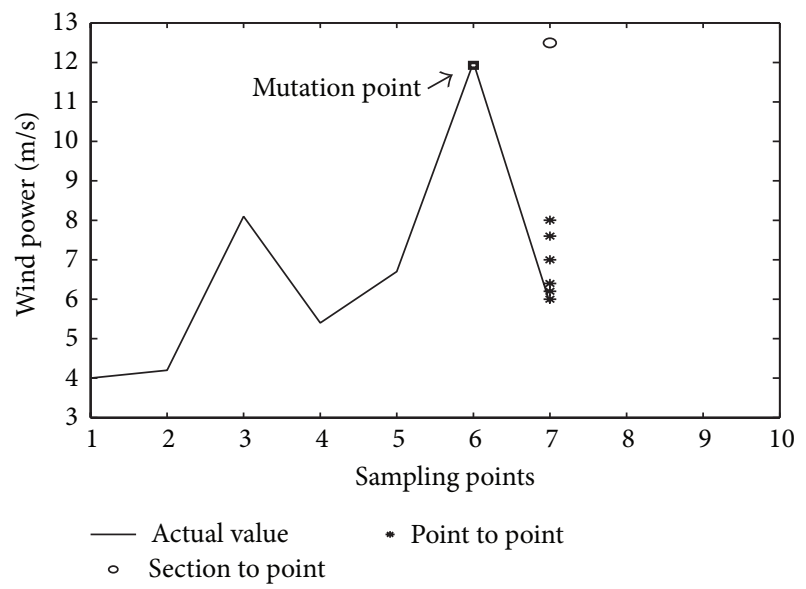

FIGURE 12: Prediction comparison between section to point and point to point.

$r_{\alpha \beta}$ means similarity coefficient of current sequence and historical sequence. It should be readjusted if results are negative:

$$
r_{\alpha \beta}^{\prime}=\frac{r_{x y}-m}{M-m}
$$

In formula (13), $m=\min r_{\alpha \beta} ; M=\max r_{\alpha \beta}$.

By comparing the number of $r_{\alpha \beta}^{\prime}$, the sequence which has a maximum value is determined as the historical sequence $Y_{n}$. 


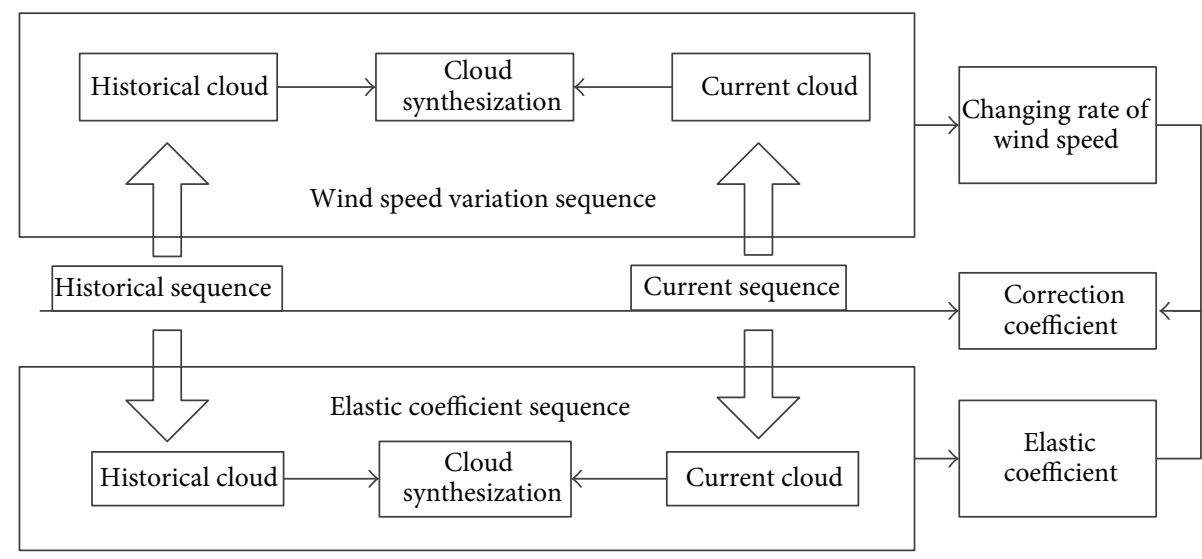

FIGURE 13: Step of "section to point" correction.

(3) Three characteristic numbers (Ex, En, He) are generated by cloud synthesization.

(4) Change rate of the wind speed and the elastic coefficient based on cloud generator forecasted, which are used to obtain the value of the correction coefficient:

$$
C=Q_{t}^{\prime} \times K_{t}^{\prime} \text {. }
$$

In formula (14), $K_{t}^{\prime}$ means forecasted value of the elastic coefficient and $Q_{t}^{\prime}$ means forecasted value of the wind speed change rate.

(5) The forecasted value of wind power can be carried out through multiplying the wind power eigenvalue by the correction coefficient.

\section{Case Study}

In order to verify the effectiveness of forecasting model proposed in this paper the wind speed and wind power data for daily load forecasting are provided by a wind power station in Inner Mongolia with a period of 60 days in 2013. Among the data, the first 30 days are used for history database matching, and the last 30 days are taken as forecasted comparative data. Figure 14 shows the data of the wind speed and wind power values in the first 30 days.

Step 1. Seven qualitative clouds are obtained after cloud transformation and concept abstracting on the basis of the wind power data in 2013. Its eigenvalues are shown in Table 1.

Table 1 also can be depicted by Figure 15 .

Eight qualitative clouds are obtained after cloud transformation and concept abstracting based on the wind speed data in 2013, whose eigenvalues are shown in Table 2.

Table 2 also can be depicted by Figure 16.

The a priori algorithm is applied to mining the association rules between historical wind speed and wind power. 8 rules that can satisfy the constraint of the minimum support threshold and the minimum confidence threshold are determined. The rules are shown in Table 3.

Take average wind speed $6.2(\mathrm{~m} / \mathrm{s})$ of the 30 th day as input value; then the corresponding 8 th class qualitative

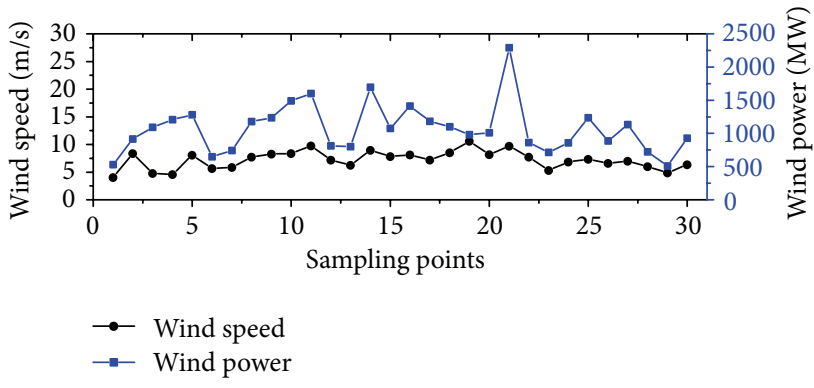

FIGURE 14: Historical data for wind speed and power.

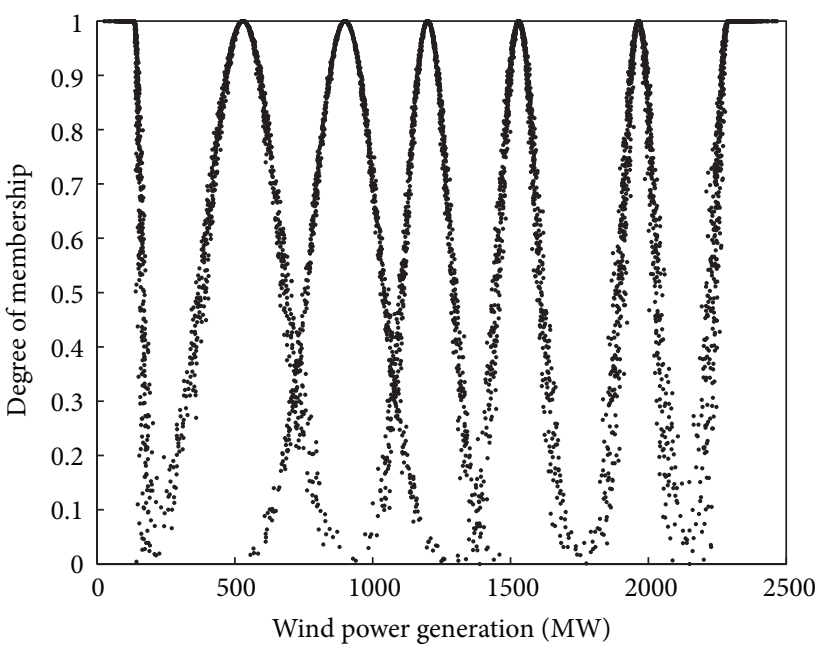

FIGURE 15: Cloud chart after the wind power concept retrieval.

cloud would be activated. The cloud which has the highest membership degree is taken as the forward rule. Also, Wind speed $6.2(\mathrm{~m} / \mathrm{s})$ corresponding to the qualitative concept of "Slightly fast 2" will be drawn. According to the 5th rule, wind power cloud which is named "high" is taken as the backward rule. Current period wind power eigenvalue can be calculated based on mining associated rules, and the value is 1238.4 (MW). This wind power eigenvalue also can be taken 
TABLE 1: Eigenvalue of the qualitative concept of wind power.

\begin{tabular}{lccc}
\hline & Ex & En & He \\
\hline Rather low & 135 & 25 & 7.1 \\
Low & 530 & 132 & 9.3 \\
Slightly low & 900 & 121 & 5.2 \\
Moderate & 1200 & 80 & 6.2 \\
Slightly high & 1530 & 70 & 8.5 \\
High & 1965 & 60 & 10.2 \\
Rather high & 2290 & 50 & 12.3 \\
\hline
\end{tabular}

TABLE 2: Eigenvalue of the qualitative concept of wind speed.

\begin{tabular}{lccc}
\hline & Ex & En & He \\
\hline Rather slow & 1.2 & 0.33 & 0.02 \\
Slow & 2.3 & 0.2 & 0.03 \\
Slightly slow & 3.2 & 0.3 & 0.06 \\
Moderate 1 & 4.1 & 0.25 & 0.04 \\
Moderate 2 & 5.0 & 0.32 & 0.03 \\
Slightly fast & 6.2 & 0.23 & 0.03 \\
Fast & 7.4 & 0.27 & 0.04 \\
Rather fast & 9.0 & 0.29 & 0.05 \\
\hline
\end{tabular}

TABLE 3: Association rules after mining.

\begin{tabular}{lcc}
\hline Rules & Wind speed & Wind power \\
\hline Rule 1 & Rather slow & Rather low \\
Rule 2 & Slow & Low \\
Rule 3 & Slightly slow & Slightly low \\
Rule 4 & Moderate 1 & Moderate \\
Rule 5 & Moderate 2 & Slightly high \\
Rule 6 & Slightly fast & High \\
Rule 7 & Fast & Rather high \\
Rule 8 & Rather fast & Rather high \\
\hline
\end{tabular}

TABLE 4: Comparison table of relative error (\%).

\begin{tabular}{lccc}
\hline & Maximum & Minimum & Mean \\
\hline ARMA & 42.32 & 0.51 & 16.59 \\
Power eigenvalue & 37.84 & 0.63 & 12.87 \\
\hline
\end{tabular}

as forecasting value. Figure 17 shows forecast comparison in the coming 15 days, and Table 4 shows the comparison table of relative error.

When the wind power eigenvalue is taken as a predictive value, forecasting precision is improved slightly comparing with forecasting value by the model ARMA ("point-to-point" model). But the maximum errors of them are bigger, which means both of them cannot make effective inhibition of fluctuation at the catastrophe point. Therefore, the forecasted value at the catastrophe point should be corrected, which is the second step "section to point" correction. The steps are as follows.

Step 2. (1) According to 5-day wind speed and wind power data close to the forecasting point, the first sequence of

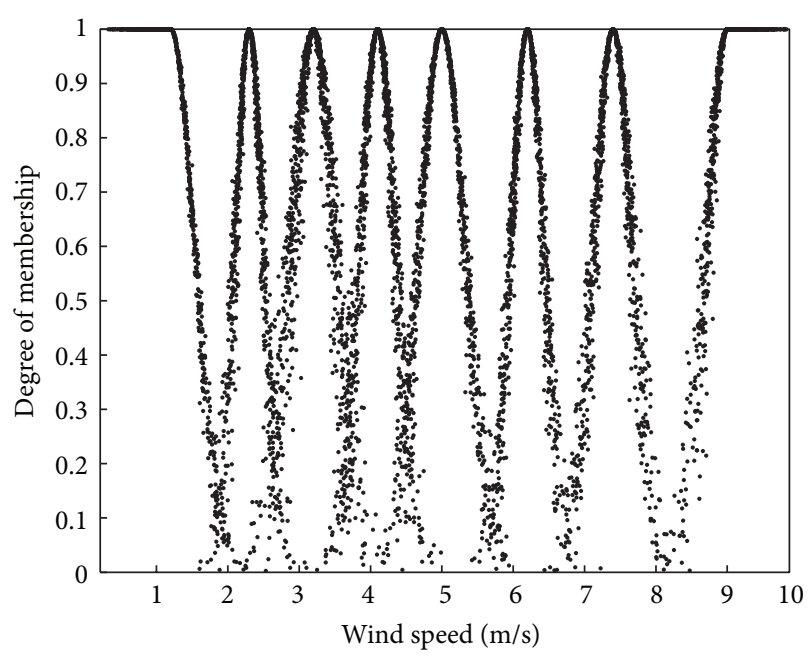

FIGURE 16: Cloud chart after the wind speed concept retrieval.

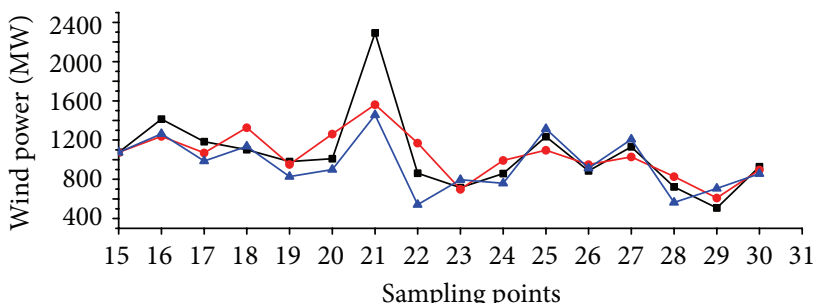

$$
\begin{aligned}
\rightarrow & \rightarrow \text { Actual } \\
\rightarrow & \text { Power eigenvalue } \\
& \text { Figure 17: Prediction comparison chart. }
\end{aligned}
$$

the elasticity coefficient can be concluded as $\alpha$. Then match $\beta$ with the highest similarity with $\alpha$ in each sequence in the first 30 days:

$$
\begin{aligned}
& \alpha=\{-0.4291,-0.0419,0.7656,-0.3008,0.0430,0.3106\}, \\
& \beta=\{-0.2032,-0.3086,0.2780,0.0774,-0.1009,0.0604\} .
\end{aligned}
$$

These two sequences above can obtain two clouds by backward cloud generator. Then, they are synthesized to produce new forecasting clouds. By setting up rules generator, it can be predicted that the elastic coefficient is 0.67 . Similarly, forecasting value of the wind speed change rate is 1.65.

(2) According to the change rate of the wind speed and the elastic coefficient of forecasting value, correction coefficient is 1.12. Finally, the forecasting value of corrected power is 1387.0 (MW). The result of the first step is shown in Figure 18, and the Table 5 shows the comparison table of relative error.

The average relative error and the maximum relative error are reduced after being corrected, and the forecasting accuracy has improved. Particularly for catastrophe point forecasting, the volatility of forecasting value is reduced, which is of great importance for wind power grid security. Figure 19 shows the simulation results in a fluctuated period. 


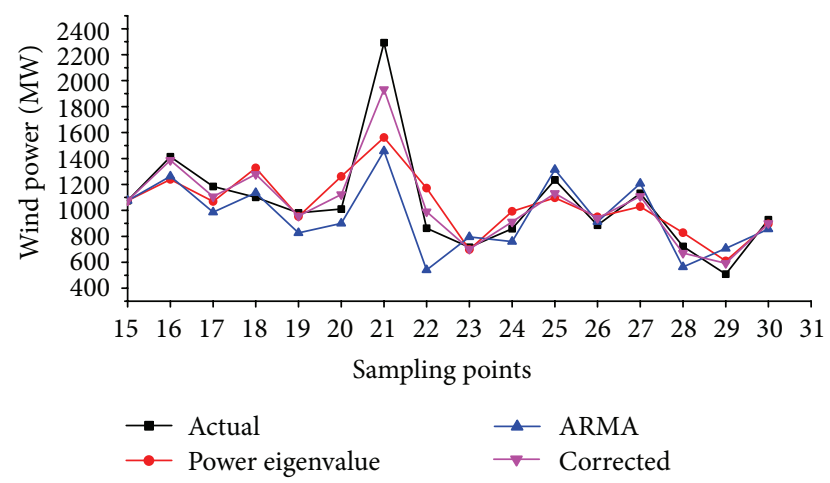

FIGURE 18: Prediction comparison after correction.

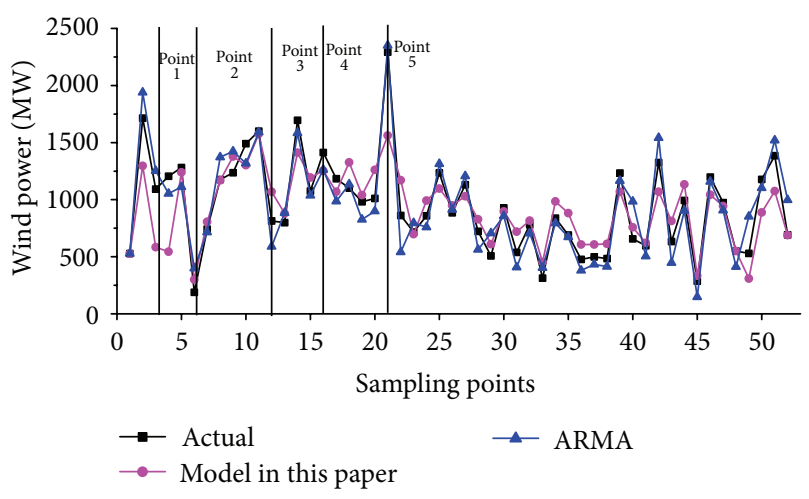

FIgURE 19: Comparison chart in mutation points.

TABLE 5: Comparison table of relative error after correction (\%).

\begin{tabular}{lccc}
\hline & Maximum & Minimum & Mean \\
\hline ARMA & 42.32 & 0.51 & 16.59 \\
Power eigenvalue & 37.84 & 0.62 & 12.87 \\
After correction & 26.21 & 0.32 & 10.87 \\
\hline
\end{tabular}

TABLE 6: Comparison table of relative error in mutation points (\%).

\begin{tabular}{lccccc}
\hline & 1 & 2 & 3 & 4 & 5 \\
\hline ARMA & 42.32 & 39.21 & 32.42 & 36.23 & 33.24 \\
Model in this paper & 14.21 & 25.32 & 26.21 & 25.23 & 12.57 \\
\hline
\end{tabular}

As can be seen from Table 6, model presented in this paper has advantages in decreasing significant error and average error, especially in catastrophe point.

\section{Conclusion}

As a "point to point" forecasting model, forecasting results of ARMA are subject to the trend of adjacent time. Moreover, huge error occurs when encountering wind speed catastrophe points. Compared with ARMA, the average relative error of this model is reduced by $3.72 \%$ in terms of prediction accuracy. The improvement of prediction accuracy is shown in the following two aspects.
First, it realizes the transformation between quantitative data and qualitative data based on cloud theory. And it builds an accurate correlation between wind speed and wind power, which reduces the average relative error by $3.72 \%$.

Second, catastrophe points forecasting realizes the "section to point" correction and the current forecasting correction by cloud synthesization and historical data. It reduces the trend inertia at catastrophe points and reduces the average relative error by $2 \%$. Particularly at catastrophe points, the reduction can reach up to $11.63 \%$.

Model proposed in this paper will improve the accuracy of prediction as historical data continuously accumulates. Given more historical data, it will be capable of attaining sequences with higher similarity from adjacent data in a broader space-time. Due to limited data in this paper, cloud reasoning and sequence analyzing are only applied to wind speed data which has significant influence on wind power. If meteorological data are added, the prediction accuracy could be further improved.

\section{Conflict of Interests}

The authors declare that there is no conflict of interests regarding the publication of this paper.

\section{Acknowledgments}

This work was financially supported by the National Natural Science Foundation of China (71401055). And the authors would like to thank all projects partners for their contribution.

\section{References}

[1] A. M. Foley, P. G. Leahy, A. Marvuglia, and E. J. McKeogh, "Current methods and advances in forecasting of wind power generation," Renewable Energy, vol. 37, no. 1, pp. 1-8, 2012.

[2] N. Haouas and P. R. Bertrand, "Wind farm power forecasting," Mathematical Problems in Engineering, vol. 2013, Article ID 163565, 5 pages, 2013.

[3] L. C. Cradden, F. Restuccia, S. L. Hawkins et al., "Consideration of wind speed variability in creating a regional aggregate wind power time series," Resources, vol. 3, no. 1, pp. 215-234, 2014.

[4] S. S. Soman, H. Zareipour, O. Malik, and P. Mandal, "A review of wind power and wind speed forecasting methods with different time horizons," in Proceedings of the North American Power Symposium (NAPS '10), pp. 1-8, Arlington, Va, USA, September 2010.

[5] G. Sideratos and N. D. Hatziargyriou, "An advanced statistical method for wind power forecasting," IEEE Transactions on Power Systems, vol. 22, no. 1, pp. 258-265, 2007.

[6] M. Ranaboldo, G. Giebel, and B. Codina, "Implementation of a Model Output Statistics based on meteorological variable screening for short-term wind power forecast," Wind Energy, vol. 16, no. 6, pp. 811-826, 2013.

[7] W. Yu, A. Plante, S. Dyck et al., "An operational application of NWP models in a wind power forecasting demonstration experiment," Wind Engineering, vol. 38, no. 1, pp. 1-22, 2014. 
[8] A. Bossavy, R. Girard, and G. Kariniotakis, "Forecasting ramps of wind power production with numerical weather prediction ensembles," Wind Energy, vol. 16, no. 1, pp. 51-63, 2013.

[9] X.-X. Meng, C.-W. Tian, L. Dong, Y. Gao, Y. Hao, and X.-Z. Liao, "A study of grey theory used in prediction of medium and long-term wind power generation," Power System Protection and Control, vol. 39, no. 21, pp. 81-85, 2011 (Chinese).

[10] J. L. Torres, A. García, M. de Blas, and A. de Francisco, "Forecast of hourly average wind speed with ARMA models in Navarre (Spain)," Solar Energy, vol. 79, no. 1, pp. 65-77, 2005.

[11] A. Y. Alanis, L. J. Ricalde, C. Simetti, and F. Odone, "Neural model with particle swarm optimization Kalman learning for forecasting in smart grids," Mathematical Problems in Engineering, vol. 2013, Article ID 197690, 9 pages, 2013.

[12] J. P. S. Catalão, H. M. I. Pousinho, and V. M. F. Mendes, "Shortterm wind power forecasting in Portugal by neural networks and wavelet transform," Renewable Energy, vol. 36, no. 4, pp. 1245-1251, 2011.

[13] R. Zhang, G. Qi, C. Li et al., "Forecasting of load model based on typical daily load profile and BP neural network," in Proceedings of the 5th International Conference on Machine Vision (ICMV '12), vol. 8784, p. 7, International Society for Optics and Photonics, 2013.

[14] K.-C. Di, D.-Y. Li, and D. R. Li, "Cloud theory and its applications in spatial data mining and knowledge discovery," Journal of Image and Graphics A, vol. 4, no. 11, pp. 930-935, 1999.

[15] P. Lv, L. Yuan, and J. Zhang, "Cloud theory-based simulated annealing algorithm and application," Engineering Applications of Artificial Intelligence, vol. 22, no. 4-5, pp. 742-749, 2009.

[16] K. Qin, K. Xu, F. Liu, and D. Li, "Image segmentation based on histogram analysis utilizing the cloud model," Computers and Mathematics with Applications, vol. 62, no. 7, pp. 2824-2833, 2011.

[17] H. Meng, S.-L. Wang, and D.-Y. Li, "Concept extraction and concept hierarchy construction based on cloud transformation," Journal of Jilin University (Engineering and Technology Edition), vol. 40, no. 3, pp. 782-787, 2010 (Chinese).

[18] H. Chen and B. Li, "Cloud reasoning method and its application in prediction," Computer Science, vol. 38, no. 7, p. 20, 2011. 


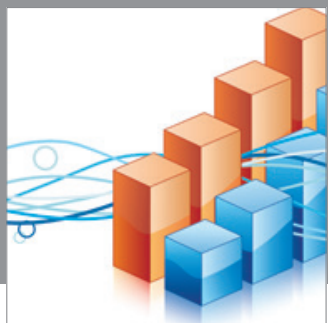

Advances in

Operations Research

mansans

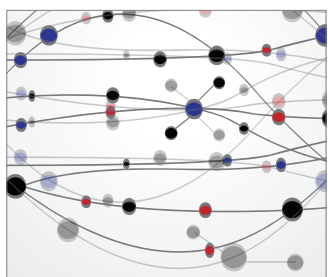

The Scientific World Journal
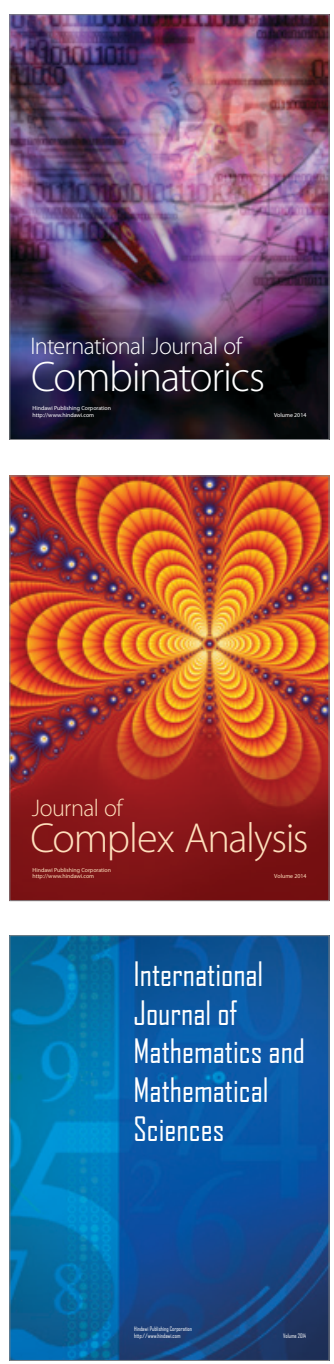
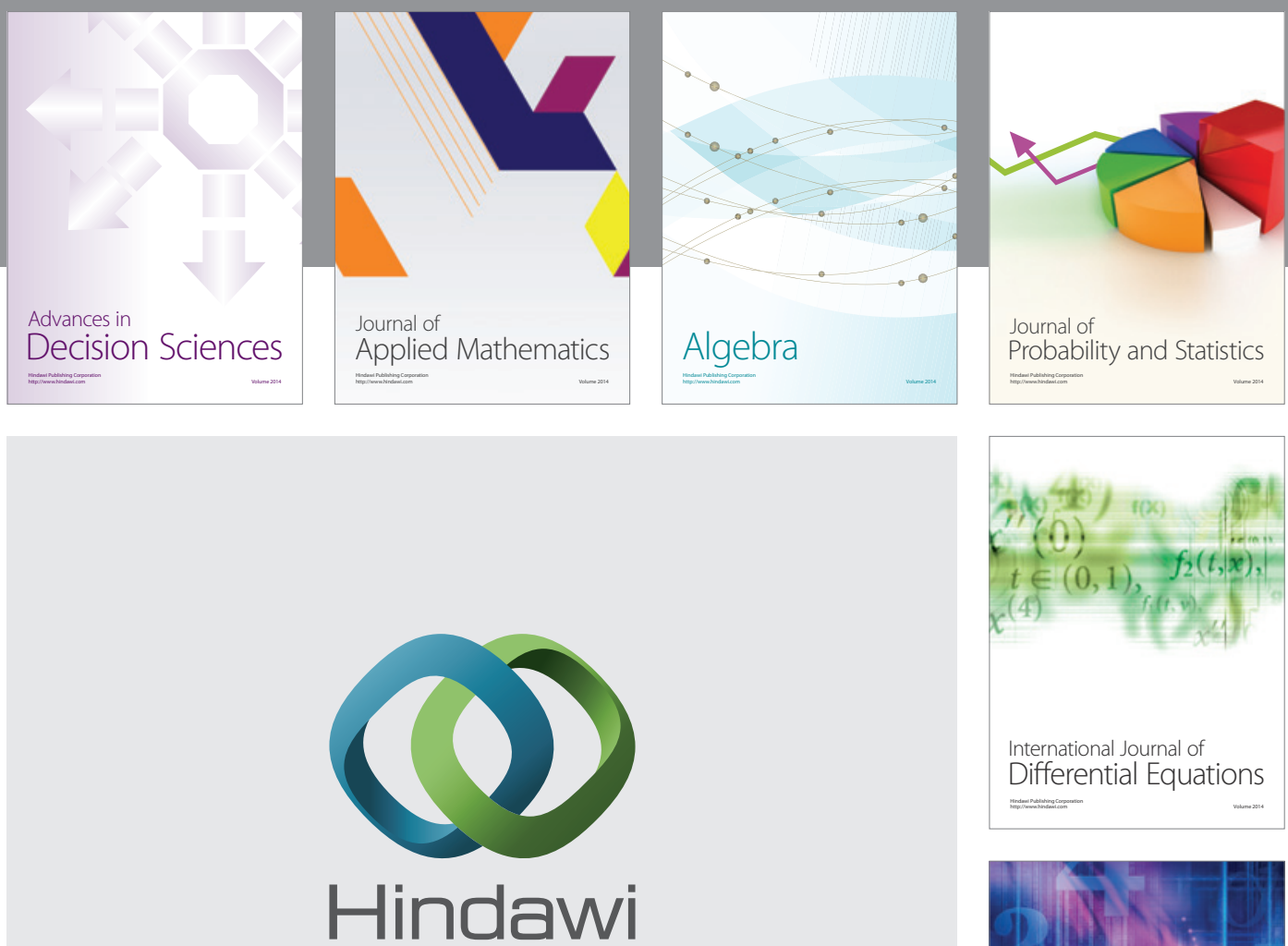

Submit your manuscripts at http://www.hindawi.com
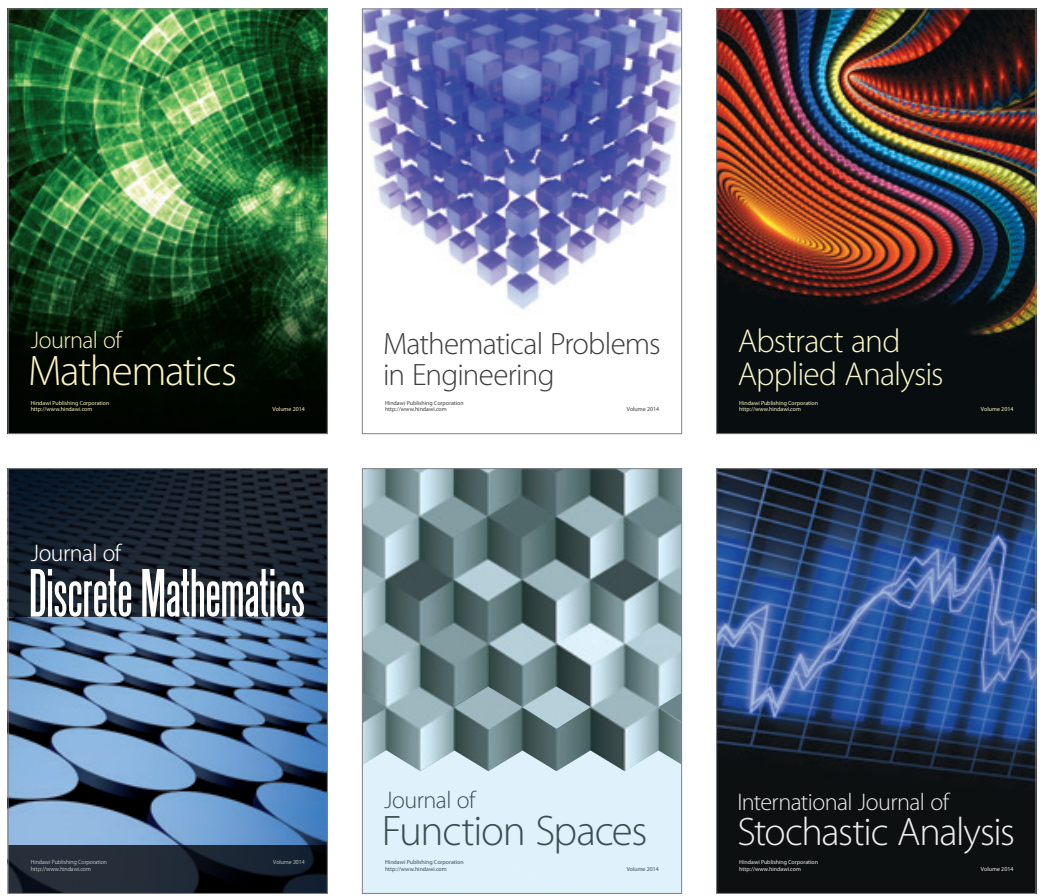

Journal of

Function Spaces

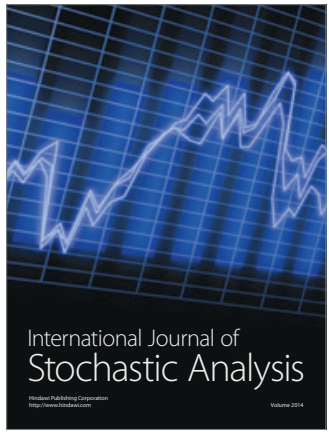

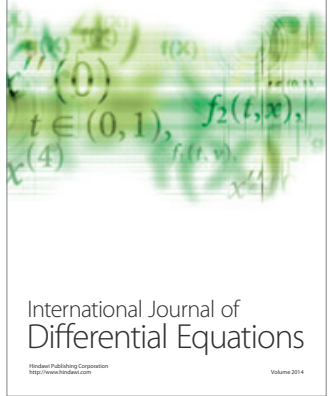
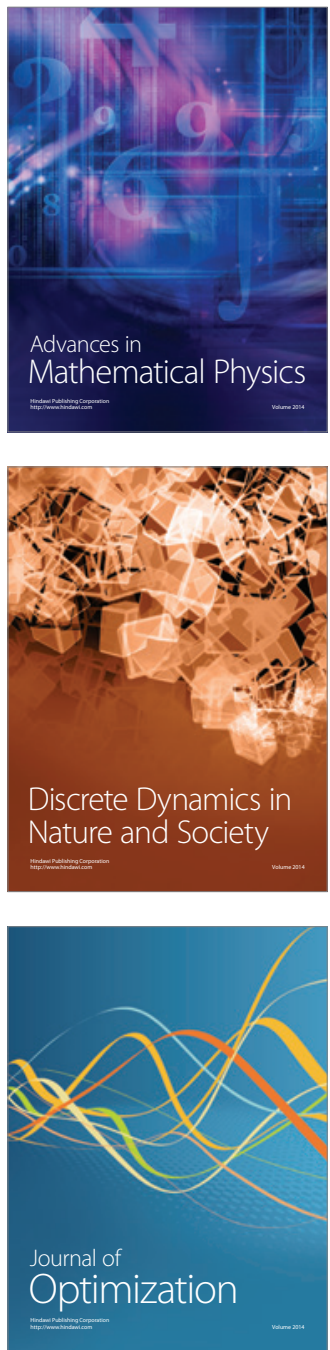